\title{
Aplicaciones biomédicas del titanio y sus aleaciones
}

F.J. GIL Y J.A. PLANELL

Dpto. de Ciencia de los Materiales e Ingeniería Metalúrgica, Universidad Politécnica de Cataluña E.T.S. Ingenieros Industriales Barcelona. Diagonal 647,08028 Barcelona.

\section{INTRODUCCION}

Hasta el siglo XVIII los materiales metálicos utilizados en implantes quirúrgicos eran fundamentalmente el oro y la plata, siendo en el siglo XIX cuando empezaron a utilizarse los aceros, y ya en este siglo aparecieron los aceros inoxidables, las aleaciones de cromo, cobalto y molibdeno, siendo en la década de los años 40 cuando se introdujeron el titanio y sus aleaciones en el campo de la medicina. Fueron Bothe, Beaton y Davenportl los que mediante la implantación en animales, observaron su excelente biocompatibilidad, comparable a la del acero inoxidable o a la del Vitallium (CoCrMo).

Su baja densidad, $4,7 \mathrm{~g} / \mathrm{cm}^{3}$ comparada con 7,9 del acero inoxidable AISI 316, 8,3 de la aleación CoCrMo y 9,2 de la CoNiCrMo, junto a sus buenas propiedades mecánicas y su excelente resistencia a la corrosión, hacen del titanio un biomaterial de sumo interés para su aplicación en implantes quirúrgicos.

En el presente trabajo se revisan las características más importantes del titanio y sus aleaciones. Después de presentar un breve resumen del concepto de biocompatibilidad, se describen fundamentalmente las aplicaciones de dichos materiales en aplicaciones ortopédicas y dentales. Aunque el titanio se utiliza también en otro tipo de aplicaciones biomédicas, tales como válvulas cardíacas (figura 1) o marcapasos, en ellas no se aprovecha todo su potencial como material estructural como en las aplicaciones que se describen en el presente trabajo.

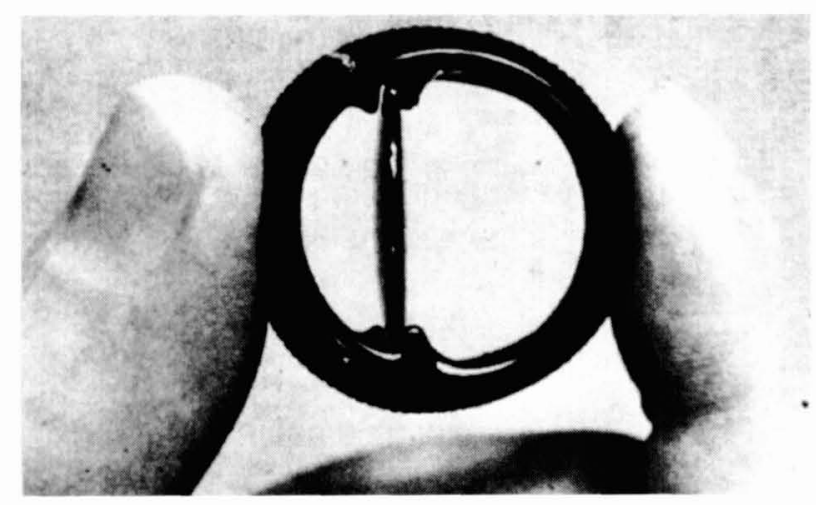

Fig. 1 Válvula cardíaca tipo disco elaborada con Titanio recubierta de carbono pirolítico

\section{EL TITANIO Y SUS ALEACIONES}

\subsection{EL TITANIO COMERCIALMENTE PURO}

El titanio es un metal que sufre una transformación alotrópica a la temperatura de $882^{\circ} \mathrm{C}$, pasando de una estructura hexagonal compacta (fase a) a una estructura más abierta cúbica centrada en el cuerpo (fase b), lo que permite la realización de tratamientos térmicos con transformación total. Hay que destacar la facilidad que tiene el titanio para disolver por sustitución o inserción otros elementos que, dependiendo del número de electrones de enlace del elemento, tienden a estabilizar alguna de las dos fases alotrópicas.

Los elementos de menos de cuatro electrones de enlace por átomo estabilizan la fase $\alpha$ y aumentan la temperatura de transformación, como puede observarse en la figura 2a. Este efecto lo producen elementos como el aluminio o el galio y los intersticiales como el oxígeno, nitrógeno y carbono. Con el aluminio, el titanio forma un compuesto intermetálico $\left(\mathrm{Ti}_{3} \mathrm{Al}\right)$ fase $\alpha_{2}$ que provoca fragilidad al material.

Los elementos de más de cuatro electrones de enlace por átomo estabilizan la fase $\beta$ y disminuyen la temperatura de transformación. Estos pueden clasificarse en dos grupos:

- $\beta$-isomorfos como el molibdeno, wolframio y vanadio, entre otros, que forman diagramas de equilibrio como el de la figura $2 \mathrm{~b}$.

- $\beta$-eutectoides como el cobre, manganeso, hierro, niquel, cobalto y el intersticial hidrógeno, los cuales

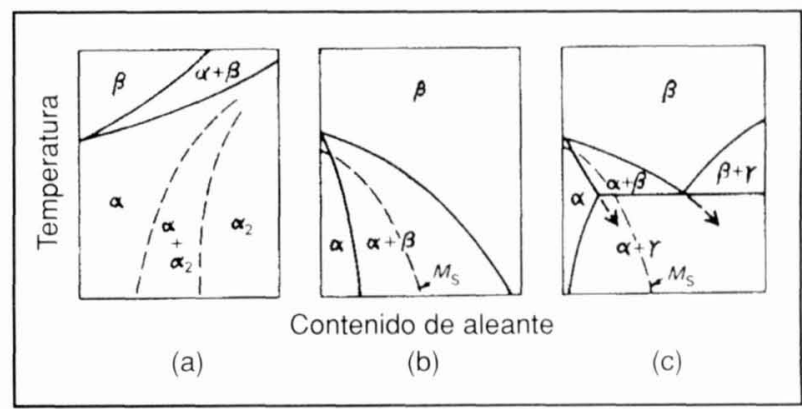

Fig. 2. Tipos de diagramas de equilibrio para aleaciones de Titanio (2). 
TABLA 1

PROPIEDADES MECANICAS DEL TITANIO PURO PARA IMPLANTES QUIRURGICOS (4)

\begin{tabular}{lcccc}
\hline Propiedades & Grado 1 & Grado 2 & Grado 3 & Grado 4 \\
\hline Carga de rotura (MPa) & 240 & 345 & 450 & 550 \\
Límite elástico (0.2\%) (MPa) & 170 & 275 & 380 & 485 \\
Elongación (\%) & 24 & 20 & 18 & 15 \\
Estricción (\%) & 30 & 30 & 30 & 25 \\
\hline
\end{tabular}

favorecen la transformación eutectoide $\beta-\alpha+$, aunque ésta no suele presentarse, por lo que en la práctica, se comportan como los $\beta$-isomorfos (figura $2 c$ ) $(2,3$.)

Existen cuatro grados de titanio sin alear normalizados para implantes quirúrgicos, dependiendo del contenido de impurezas (oxígeno, nitrógeno, carbono, hidrógeno y hierro) que son las que controlan sus propiedades mecánicas y que se expresan en la Tabla 1.

La resistencia del titanio y sus aleaciones es en general inferior a las de acero inoxidable AISI 316 y a las de las aleaciones base cobalto, pero en relación a su resistencia específica (resistencia/densidad), las aleaciones de titanio con superiores a las demás.

Otra de las ventajas del titanio en aplicaciones biomédicas, frente al acero inoxidable y a las aleaciones base cobalto, es que su módulo de Young es de $110 \mathrm{GPa}$ frente a los $200 \mathrm{GPa}$ y los $220 \mathrm{GPa}$ de las aleaciones anteriores respectivamente. Siendo el módulo elástico del hueso de $20 \mathrm{GPa}$, se comprende que el titanio, es más elásticamente compatible con el tejido natural que las otras dos aleaciones. Sin embargo, el titanio tiene una pobre resistencia a cizalladura, por lo que no es adecuado para tornillos óseos y clavos.

El titanio y sus aleaciones poseen una excelente resistencia a la corrosión, ya que la rápida reacción del titanio con el oxígeno, produce una fina capa superficial de óxido impermeable y por lo tanto protectora. Este comportamiento, que tiene también lugar en el aluminio, es más eficiente en el titanio, dando lugar a una resistencia tanto a la oxidación como a la corrosión superiores. Todo ello justifica su uso en la industria química y en ambientes altamente corrosivos, y en particular hace del titanio y sus aleaciones los metales más adecuados para la fabricación de implantes quirúrgicos (5-7).

\subsection{LA ALEACION TI-6A1-4V}

La aleación Ti-6A1-4V es la más utilizada industrialmente de entre todas las aleaciones de titanio y a ella se dedica la mitad de la producción de titanio metal. Esto se debe al excelente balance entre sus propiedades mecánicas, su resistencia a la corrosión, su buen comportamiento a temperaturas elevadas, debiendo destacarse también su capacidad para ser trabajado mecánicamente y de modificar sus propiedades mediante tratamientos térmicos.

La aleación Ti-6A1-4V es una aleación de tipo $\alpha-\beta$. Este tipo de aleaciones se han desarrollado por la mala

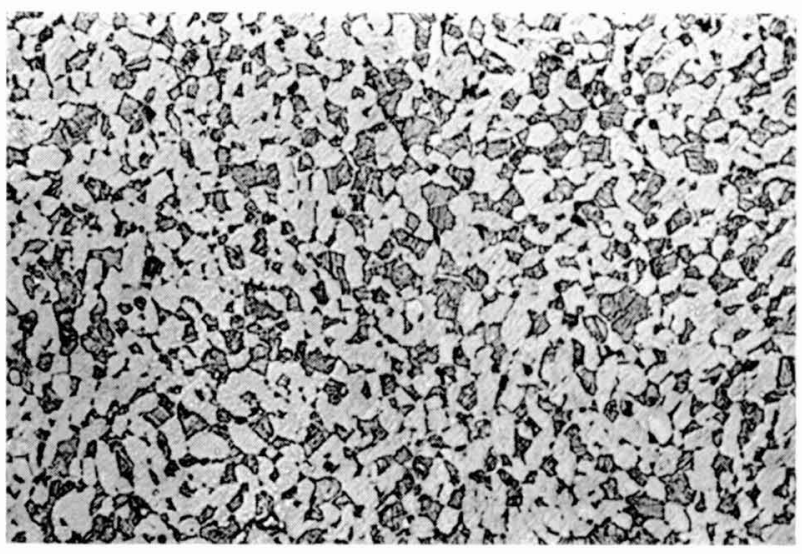

Fig. 3. Estructura «mill-annealed».

forjabilidad de las de tipo a y super-a, por su baja resistencia en frío y por su fragilidad si se intenta aumentar su resistencia mediante el aumento del contenido de elementos de aleación (2).

Si esta aleación se calienta en la regresión $\alpha-\beta$, se produce una microestructura formada por granos equiaxiales de fase $\alpha$ en una matriz de fase $\beta$. Al enfriar lentamente, la fase $\beta$ transforma a $\alpha$ con una morfología que se denomina «mill-annealed», consistente en granos equiaxiales de fase $\alpha$ y placas de Widmanstatten de fase a rodeadas de fase $\beta$ no transformada $(2,8)$. Esta microestructura puede observarse en la figura 3 .

Cuando el tratamiento térmico que se realiza sobrepasa la temperatura del $\beta$-transus, que para esta aleación está alrededor de los $1030^{\circ} \mathrm{C}$, la estructura es totalmente $\beta$, produciéndose un rápido crecimiento de grano. Al enfriar lentamente, se produce la transformación adoptando la fase a la morfología de placas de Widmanstatten, rodeadas de fase $\beta$ que no ha transformado. El tamaño de estas placas de Widmanstatten depende de la temperatura y tiempo de mantenimiento del tratamiento térmico y sobre todo, de la velocidad de enfriamiento. La microestructura, denominada de tipo «cesto», corresponde a la que se observa en la figura 4 , y es característica de un enfriamiento lento $(9,10)$.

Esta aleación es susceptible de transformar martensíticamente, si el enfriamiento desde la fase $\beta$ es rápido, obteniéndose morfologías aciculares de martensita $\alpha$ ', como se puede apreciar en la figura 5. Esta martensita puede sufrir tratamientos de revenido $\left(\alpha^{\prime} \alpha+\beta\right)$ que provocan una fina precipitación de fase a alrededor de las placas martensíticas aumentando así de forma considerable la dureza de la aleación $(11,12)$. 
TABLA 2.

CARACTERISTICAS MECANICAS A TRACCION DE LA ALEACION Ti-6A1-4V

\begin{tabular}{lcccc}
\hline Estructura & $\begin{array}{c}\text { Límite } \\
\text { elástico } \\
0.2 \%(\mathrm{MPa})\end{array}$ & $\begin{array}{c}\text { Resistencia } \\
\text { a tracción } \\
(\mathrm{MPa})\end{array}$ & $\begin{array}{c}\text { Elongación } \\
(\%)\end{array}$ & $\begin{array}{c}\text { Estricción } \\
(\%)\end{array}$ \\
\hline Mill-annealed & 880 & 945 & 18 & 34 \\
Widmanstatten & 750 & 885 & 15 & 18 \\
\hline
\end{tabular}

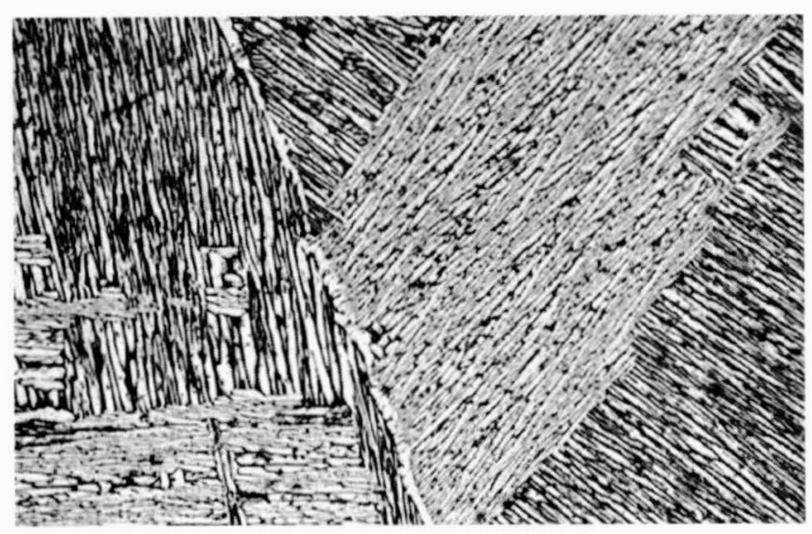

Fig. 4. Estructura obtenida después de tratamiento térmico. (1050 ${ }^{\circ}$ durante 1 hora y enfriada en el interior del horno).

La estructura «mill-annealed» presenta un límite elástico, resistencia a la tracción, alargamiento a la rotura y estricción superiores a los que presenta la estructura de tipo «cesto», obtenida esta última por enfriamiento lento desde la región $\beta$, tal como se indica en la Tabla 2 (13).

Sin embargo, está bien demostrado que la tenacidad a fractura y la resistencia a fatiga son superiores, y la velocidad de propagación de grietas inferior en la estructura Widmanstatten en relación a la «mill-annealed». En estudios recientes referentes a la nucleación de grietas (13-17) se observa que éstas se producen tanto a partir de bandas de deslizamiento en la fase $\alpha$, equiáxica o de Widmanstatten, como por descohesión en las intercaras $\alpha-\beta$, existiendo también la posibilidad en la estructura de Widmanstatten, de grietas que se nuclean a través de las intercaras $\alpha-\beta(13,18)$. Cabe señalar, así mismo, la importancia de la textura tanto en la nucleación como en la propagación de grietas por fatiga. En general se puede decir que en la estructura en placas de
Widmanstatten la propagación de grietas es muy sensible a la microestructura (13-18). Ello es lo que explica la mayor tenacidad a fractura y la menor velocidad en la propagación de grietas.

Se han realizado estudios de corresión fatiga $(19,20)$, ya que la mayoría de los implantes están sujetos a cargas dinámicas en medios corrosivos. La corrosión hace decrecer el límite de fatiga aunque la repasivación del metal se produce con suficiente rapidez como para definirse un nuevo límite. En la figura 6 se muestra como el límite de fatiga disminuye de 60 a $60 \mathrm{MPa}$ para esta aleación (21). Se puede observar en la Tabla 3, como en todos los casos el límite de fatiga decrece.

\subsection{ALEACIONES DE TITANIO CON MEMORIA DE FORMA}

Las aleaciones de Niquel-Titanio con memoria de forma son de gran interés, ya que además de las propiedades físicas conocidas en los materiales metálicos convencionales, presentan otras como son el efecto memoria de forma y la superelasticidad. Estas propiedades son también de interés en el campo de la medicina.

Para que se produzcan estas inusuales propiedades es necesario para la aleación presente una fase austenítica suceptible de una transformación martensítica termoelástica, es decir, que los cambios de forma y volumen producidas en la transformación sean de tipo elástico, lo que lleva consigo una transformación reversible. En la retransformación (martensita austenita), la fase austenítica no necesita renuclearse en el interior de la martensita sino que las placas de martensita van desapareciendo por movimiento interfacial de las placas en sentido contrario al de crecimiento hasta que todo el material vuelve a encontrarse en fase austenítica. Si por el contrario, las energías puestas en juego hubiesen producido deformación plástica, la transformación no sería termoelásti-

TABLA 3.

LIMITES DE FATIGA PARA ALGUNOS METALES USADOS EN IMPLANTES QUIRURGICOS (21-23)

\begin{tabular}{lcc}
\hline & \multicolumn{2}{c}{ Límite de fatiga (MPa) } \\
& Aire & Medio fisiológico \\
\hline Acero Inoxidable 316 & $260-280$ & $230-270$ \\
Aleación CoCrMo & 310 & $240-280$ \\
Ti-6A1-4V & 600 & 500 \\
Ti comercial puro & 300 & 240 \\
\hline
\end{tabular}




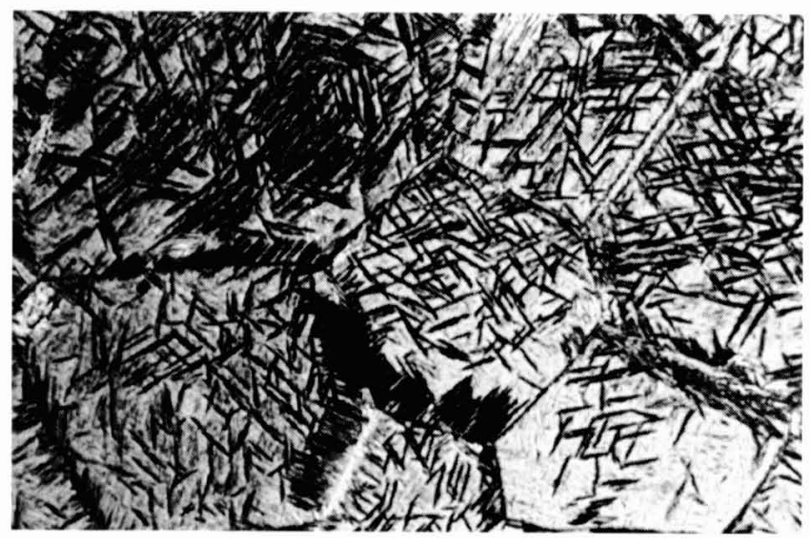

Fig. 5. Estructura martensítica a'. $\left(1050{ }^{\circ} \mathrm{C}\right.$ durante 1 hora y temple en agua).

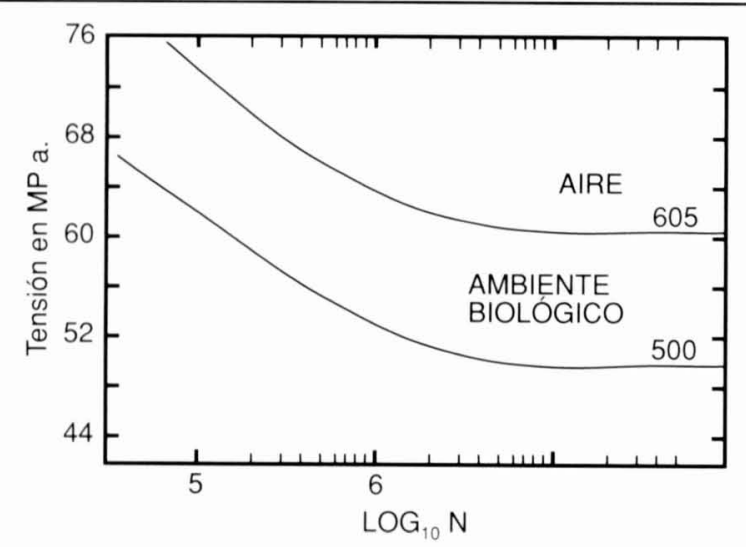

Fig. 6. Resultados de corrosión-fatiga en aire y en ambiente biológico (21).

ca y la fase austenítica debería ser nucleada de nuevo, como ocurre en el caso de la mayoría de aleaciones base hierro. La plasticidad lleva asociada la irreversibilidad y una gran histéresis térmica entre la tranformación directa $(\mathrm{A} \rightarrow \mathrm{M})$ e inversa $(\mathrm{M} \rightarrow \mathrm{A})$, como las que acaecen en los aceros, a diferencia de las transformacioes martensíticas termoelásticas, en que la histéresis es pequeña $\left(\approx 10^{\circ} \mathrm{C}\right)(24,25)$.

El efecto memoria de forma consiste en que el material con estructura martensítica se puede deformar plásticamente por debajo de la temperatura $\mathrm{A}_{\mathrm{s}}$ (temperatua de comienzo de la retransformación), pudiendo recobrarse su forma original por un simple calentamiento a temperatura por encima de $\mathrm{A}_{\mathrm{f}}$ (temperatura final de la retransformación) (25).

El comportamiento super o pseudoelástico se caracteriza por la posibilidad de obtener grandes deformaciones elásticas debidas a la aparición de placas martensítićas inducidas por tensión y además por la reorientación de dichas placas.

La curva típica de tensión-deformación de la aleación niquel-titanio (austenita a temperatura ambiente) viene ilustrada en la figura 7. En el punto A de la representación gráfica (límite elástico de la fase austenita), comienza a producirse martensita inducida por tensión (S.I.M.) progresivamente hasta $\mathrm{B}$, produciéndose la deformación

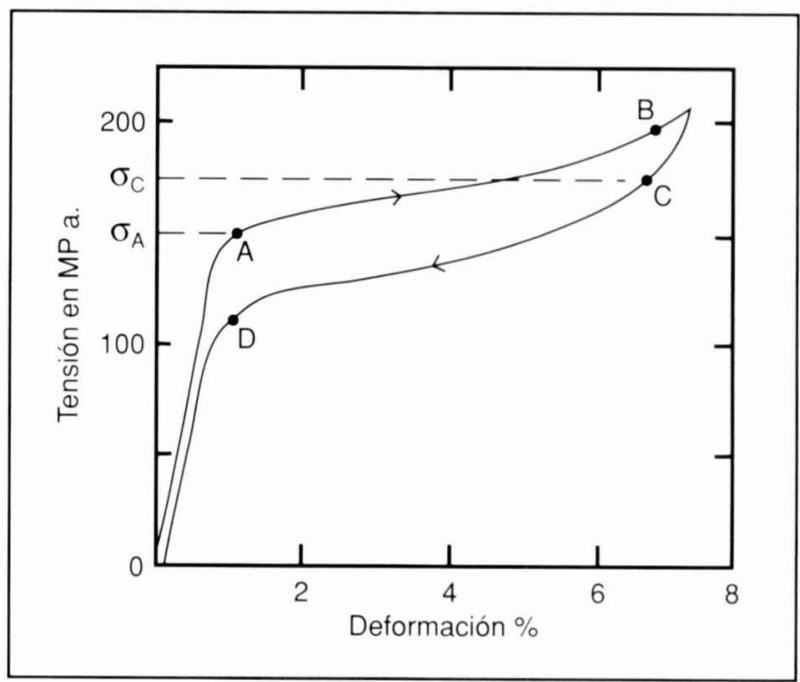

Fig. 7. Curva tensión-deformación para una aleación Ni-Tri.

en el campo elástico de la martensita. Al descargar se produce una recuperación elástica de la martensita hasta C y seguidamente se produce la retransformación en el intervalo $\mathrm{CD}$, a partir de $\mathrm{D}$ se produce la recuperación elástica de la fase austenítica. Si se sigue deformando por encima del punto B se entra dentro del campo elástico de la martensita, posteriormente en su campo plástico y eventualmente se alcanzaría la rotura. En las aleaciones niquel-titanio, se pueden alcanzar recuperacions superelásticas superiores al $10 \%$ de deformación en contraste, con los biomateriales metálicos convencionales en los que su campo elástico es normalmente inferior al $0.5 \%$ de deformación, como se observa en la figura 8 (26-29).

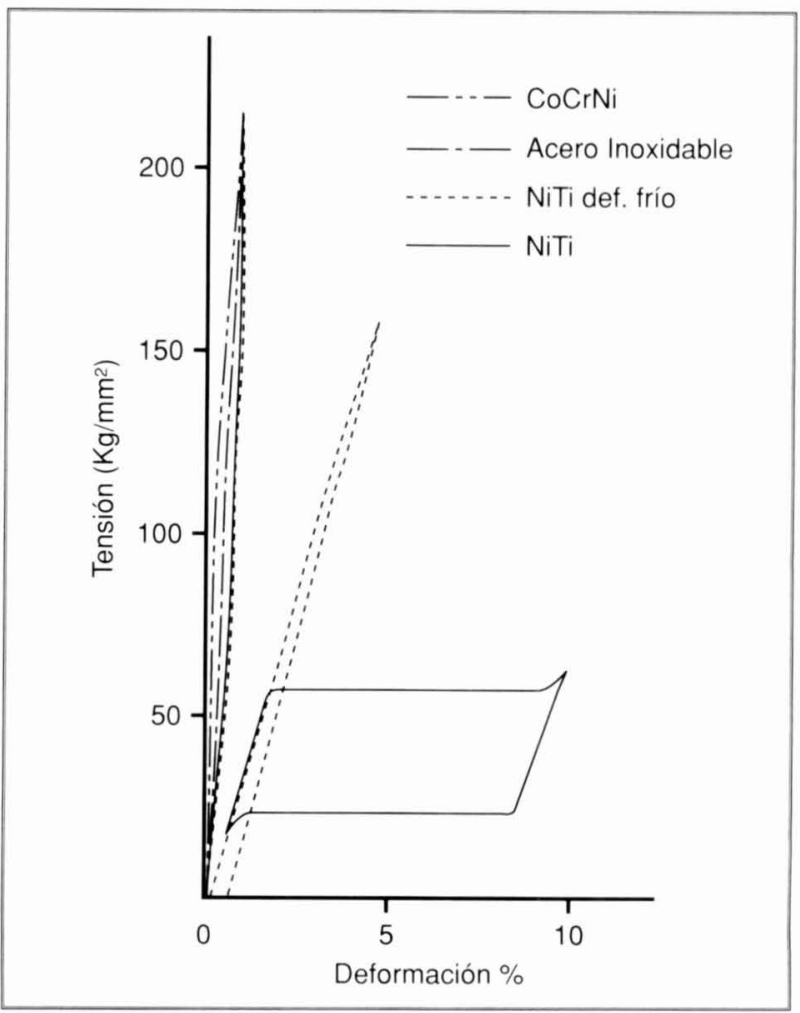

Fig. 8. CUrvas tensión-deformación de biomateriales convencionales frente a la aleación superelástica de $\mathrm{Ni}$-Tri. 


\section{BIOCOMPATIBILIDAD}

La biocompatibilidad se puede definir como la aceptabilidad biológica de materiales no vivos (biomateriales) por parte de los tejidos suscepibles de estar en contacto con ellos (30). Se puede afirmar que lo más importante de un biomaterial es que sea biocompatible. Para evaluar esta propiedad se han establecido ensayos normalizados que permiten determinar los grados de compatibilidad o en su caso de toxicidad.

Esta compatibilidad puede ser analizada desde varios niveles de interacción (30-32):

- La interacción entre el material y los tejidos.

- La reacción resultante de la degradación del material.

- Factores mecánicos (elasticidad, tenacidad, etc).

La implantación de un biomaterial lleva consigo una lesión en los tejidos vivos, reaccionando estos frente a la lesión, siendo su primera reacción una inflamación, a la que le seguirá un proceso reparador y finalmente la cicatrización de la incisión.

El proceso inflamatorio consta de dos etapas. Primero una inflamación aguda como respuesta inmediata a la lesión, produciéndose dilataciones vasculares, con aumento de permeabilidad capilar. En esta etapa las células sanguíneas fagocitan las sustancias extrañas. Posteriormente, se produce la inflamación crónica siendo una respuesta exudativa en la cual predominan los fibroblastos (células que pertenecen al tejido conectivo) acompañadas de leucocitos y macrófagos. En el proceso reparador las células que por excelencia estarán presentes serán lo fibroblastos. La cicatrización lleva consigo una exudación de leucocitos (neutrófilos) que protegen al cuerpo de microorganismos, y posteriormente aparece la fibrina portando células de tejido conectivo $(31,32)$.

La respuesta del tejido conectivo a la implantación puede producir una fibrosis mínima, que sería el caso de un sólido monolítico no tóxico, no habiendo degradación de tejidos y siendo el proceso inflamatorio de respuesta semejante al de cicatrización de una incisión. Sin embargo, la presencia del implante prolonga el proceso inflamatorio y de reparación, produciéndose desviaciones de la respuesta fibrótica mínima, lo que da lugar a la formación de una «cápsula fibrótica», debida a una reacción continuada consecuencia de una reacción fibrosa con presencia de macrófagos. También podría darse el caso de una reacción continuada consecuencia de una reacción mínima, pero muy prolongada, debida a los productos de corrosión, degradación o abrasión. En este caso se aprecian fibroblastos y fagocitos provocando la presencia de un granuloma, asociado a la presencia de edema y células gigantes $(31,32)$.

La respuesta de los tejidos duros a la implantación depende del hueso y la relación entre el hueso y el implante, pero en todos los casos tiende hacia un crecimiento o remodelación ósea (33).

El titanio y sus aleaciones presentan una excelente biocompatibilidad. Ello se debe a que dicho material es bioinerte y presenta una buena resistencia a la corrosión. Parece así mismo demostrado que la liberación de iones titanio al medio fisiológico circundante es muy bajo (34). En la actualidad existe una cierta corriente de opinión que indica que la capa de óxido que se forma en la superficie del metal al ser éste pasivado, actúa como intercara cerámica bioinerte pero que permite un excelente anclaje al tejido óseo vecino. Quizás el mayor inconveniente del titanio y sus alaciones es su baja resistencia al desgaste. Ello hace que no pueda utilizarse directamente en articulaciones. De hecho, se han empezado a utilizar técnicas de implantación iónica para mejorar el comportamiento al desgaste de cabezas femorales (35). Se ha demostrado así mismo que se puede llegar a producir desprendimiento de partículas metálicas en regiones sometidas a fricción o movimiento relativo, incluso en contacto con tejidos blandos (36).

Existe en la literatura una prevención especial sobre la biocompatibilidad del vanadio, aunque de hecho, no existe ningún estudio que demuestre que dicho elemento pueda ser nocivo cuando se encuentra combinado en la aleación Ti-6A1-4V. Es por ello que recientemente ha salido al mercado una nueva aleación $\alpha-\beta$, la Ti-6Al$7 \mathrm{Nb}$, de características muy similares a la anterior, que evita la presencia de V. Sin embargo, sólo existe una compañía que fabrique sus prótesis articulares con este nuevo material.

\section{IMPLANTES ORTOPEDICOS Y DENTALES}

\subsection{SISTEMAS DE FIJACION Y DE OSTEOSINTESIS}

Los implantes ortopédicos se utilizan fundamentalmente para la sustitución de tejidos duros, en general huesos, aunque existe un conjunto de implantes conocidos como sistemas de osteosíntesis cuya misión es la de reparar las fracturas óseas. Existe una gran variedad de sistemas de osteosíntesis que se aplican a criterio del traumatólogo según sea el tipo de fractura producida. Entre ellos cabe señalar los fijadores externos, las placas, los clavos-placa, los tornillos y los clavos intermedulares. El material más usualmente empleado para estas aplicaciones es el acero inoxidable AISI 316L. Este material no ofrece tan buenas propiedades mecánicas ni tan buena biocompatibilidad como la aleación Ti6A1-4V. Sin embargo, dado que en general los sistemas de osteosíntesis sólo se implantan temporalmente, el acero inoxidable AISI $316 \mathrm{~L}$ es suficiente para este tipo de aplicaciones, siendo también su menor precio una razón concluyente. Cabe señalar que aunque existen sistemas que utilizan materiales tipo Ti-6A1-4V o composites de fibra de grafito, no parece que su utilización pueda llegar a generalizarse debido a la razones expuestas más arriba. El futuro de estos sistemas parece estar en composites bioactivos degradables que evitarán la segunda operación quirúrgica consistente en la retirada del sistema una vez producida la consolidación ósea.

Recientemente se ha empezado a utilizar la aleación $\mathrm{Ni}$-Ti con memoria de forma para la fabricación de grapas para osteosíntesis (25). La grapa tiene inicialmente la forma que se muestra en la figura 9a. Esta se enfría en líquido fisiológico hasta $10^{\circ} \mathrm{C}$ siendo deformada plásti- 


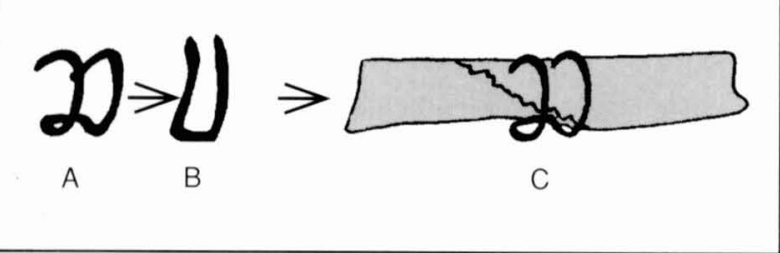

Fig. 9. Esquema de fijación de fragmentos óseos con grapas de Ni-Ti.

camente (ya que a esta temperatura su estructura es martensítica), sujetando los fragmentos óseos (figura 9b). Al calentarse hasta la temperatura corporal, la grapa tiende a recuperar la forma anterior (figura 9c), creando así durante largo tiempo una presión constnte en el límite de unión de los fragmentos óseos. La soldadura de los fragmentos siguiendo esta metodología transcurre prácticamente sin formación de callo óseo, sino «hueso contra hueso», lo que reduce considerablemente el plazo de curación.

Otro tipo de sistemas que pueden incorporar la aleación $\mathrm{Ni}$-Ti con memoria de forma son los sistemas de fijación de la columna vertebral (25). Estos sistemas se utilizan tanto en la corrección de deformaciones como para la fijación de la columna después de fractura con necesidad de consolidación de un injerto. El material empleado en la mayoría de sistemas actuales es el acero inoxidable AISI 316L. Sin embargo, la aleación con memoria de forma puede presentar ventajas tales como su capacidad para ajustarse y extenderse a medida que la columna se va enderezando. Estas aleaciones se insertan en estado plástico y con la tempertura corporal intentan retornar a su posición original realizando fuerzas constantes correctoras.

\subsection{PROTESIS ORTOPÉDICAS}

Un caso aparte son lo que se conoce como endoprótesis ortopédicas que vienen a substituir una articulación. La necesidad de su utilización reside en los problemas de artrosis articular y artritis reumatoide que acaba impidiendo el normal movimiento de una articulación o dicho movimiento se realiza con gran dolor por parte del paciente. En dicho caso, lo que se hace es una resección de la parte ósea de la articulación incorporando un sistema mecánico que venga a substituirla (37). Las prótesis de cadera y de rodilla son por este orden las de mayor aplicación, aunque existen también prótesis de hombro, codo, tobillo o muñeca. Históricamente es la prótesis de cader la más ampliamente utilizada y estudiada.

Existen dos clases de prótesis de cadera: las totales y las parciales. En las dos se secciona el cuello del fémur y se inserta el vástago de la prótesis en el interior de la cavidad medular femoral. En la prótesis total, el cartílago acetabular es retirado, colocándose en su lugar un cotillo en el que encajará la bola de la prótesis (Figura 10). Las prótesis parciales conservan el cartílago acetabular; este tipo de prótesis se coloca a personas de edad avanzada, debido a que si se coloca a personas con gran actividad física, el cartílago acetabular se deteriora con rapidez. En la figura 11 se ilustra con algunas prótesis

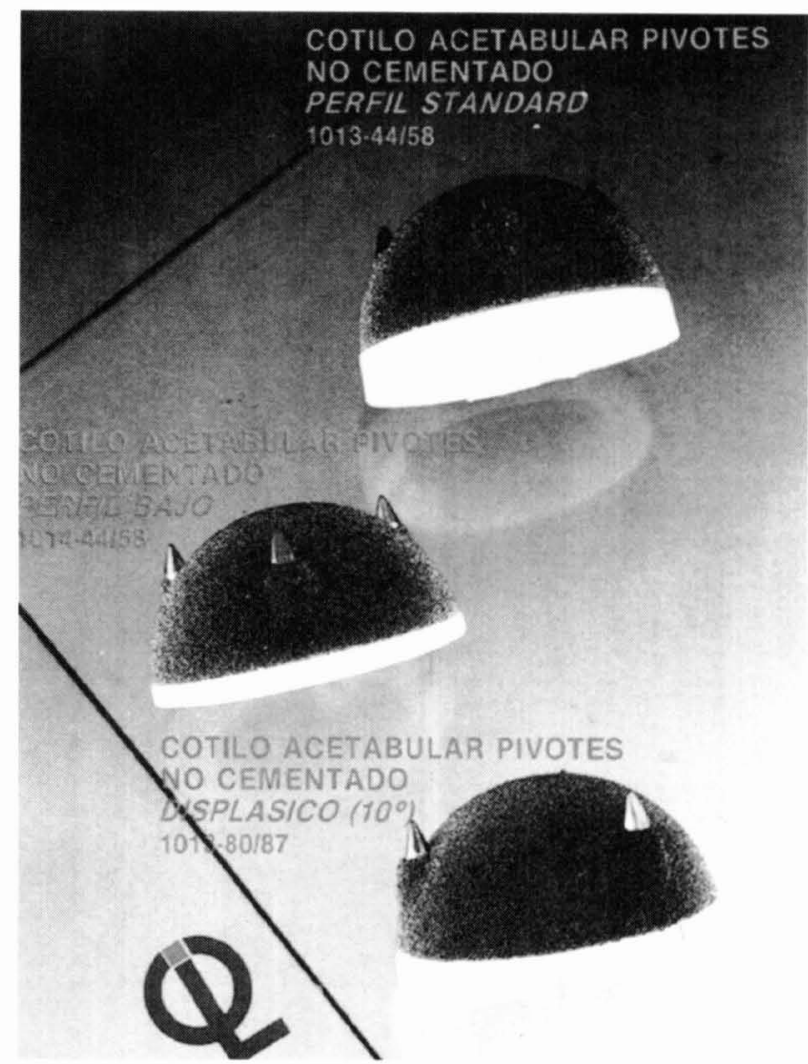

Fig. 10. Diferentes tipos de cotilos acetabulares (gentileza de Insutrias Quirúrgicas de Levante).

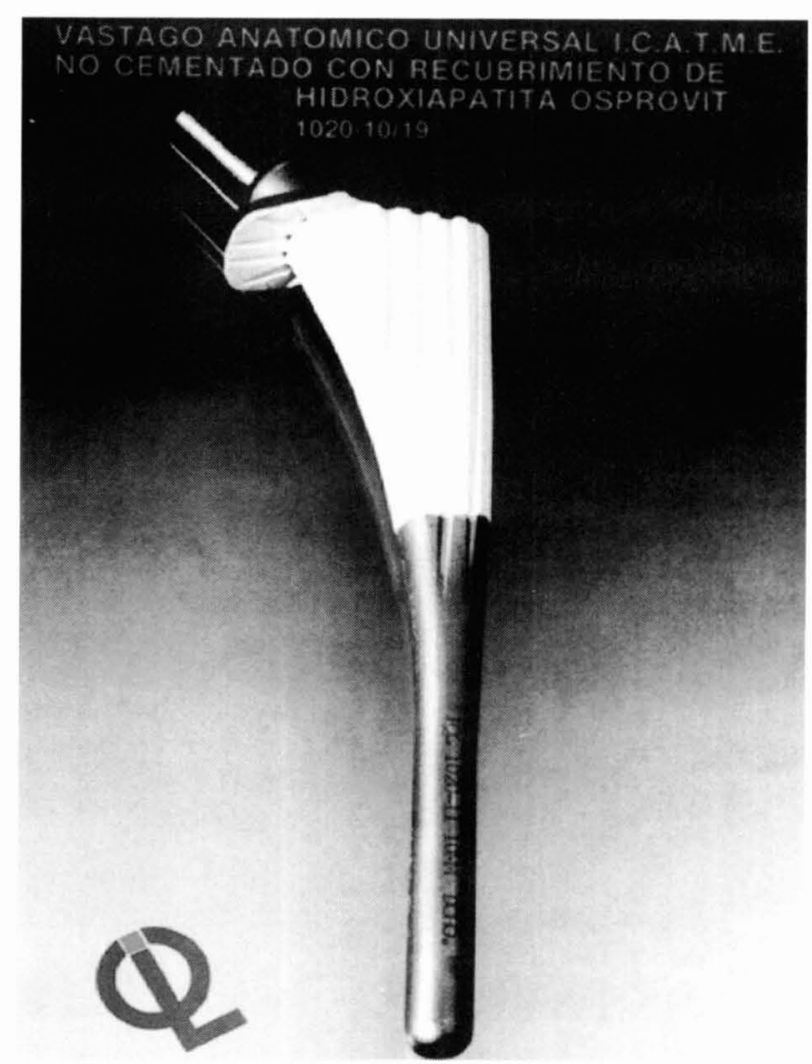

Fig. 11A. Vástago de prótesis no cementada con recubrimiento poroso de titanio. 


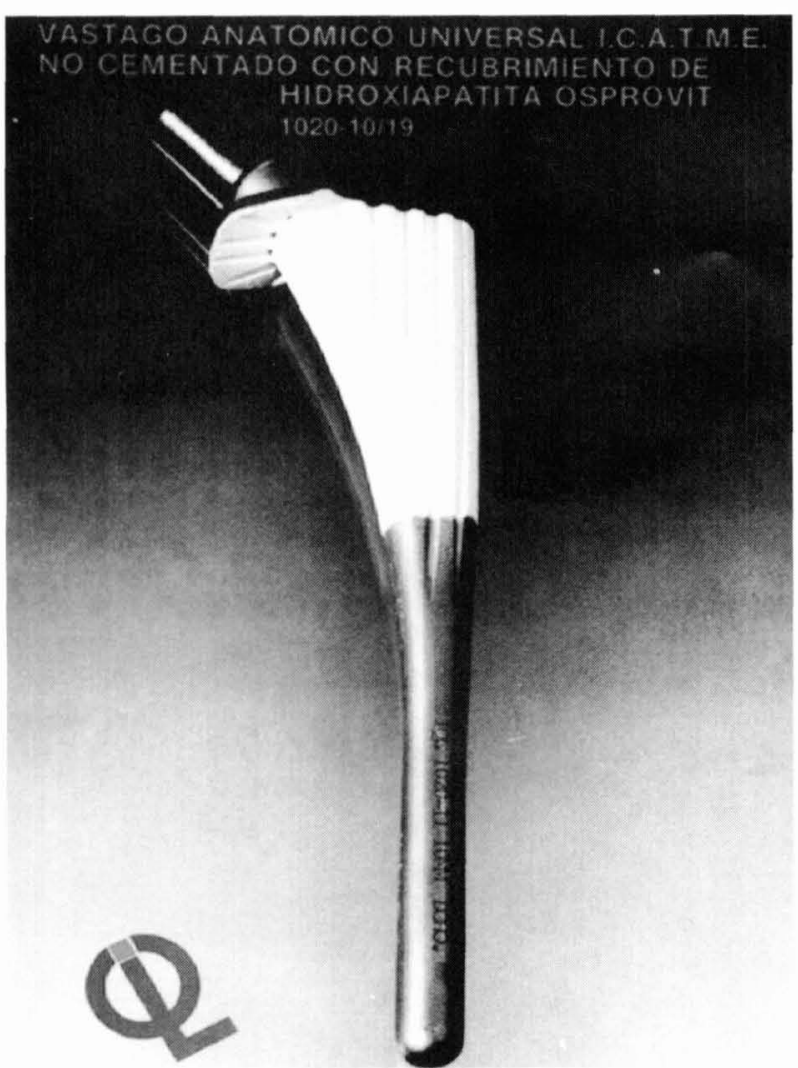

Fig. 11B. Vástago de prótesis no cementada con recubrimiento poroso de hidroxiapatito.

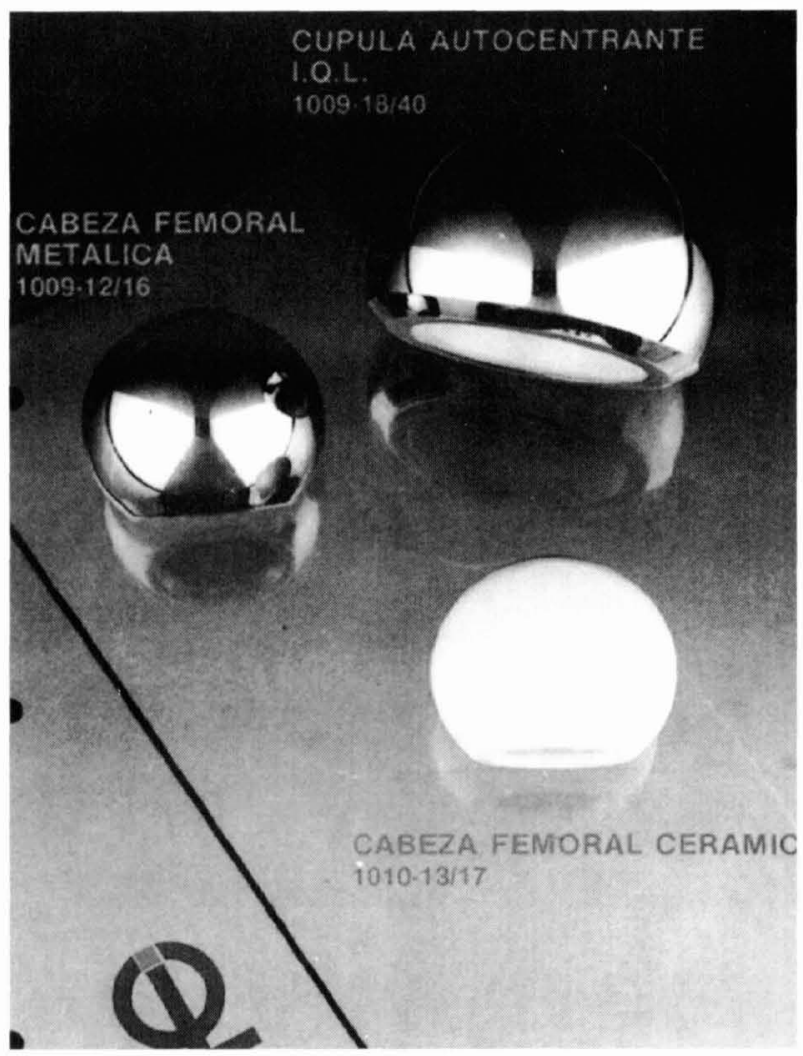

Fig. 11C. Cabezas femorales metálica y cerámica acoplables a los vástagos de la fig. 10. (Gentileza de Industrias Quirúrgicas de Levante) de cadera dentro de la gran variedad de modelos y diseños que hay en el mercado.

Para la fabricación de dichas prótesis de cadera se han utilizado históricamente diferentes materiales desde que Charnley (38) a principios de los años sesenta impuso esta técnica. En ella tanto el vástago de la prótesis •como la cúpula acetabular en la cadera van fijadas al hueso mediante un cemento óseo consistente en polimetilmetacrilato. A pesar de que la prótesis de Charney se fabrica todavía en la actualidad de acero inoxidable AISI 316L, existen en el mercado prótesis de aleaciones $\mathrm{Cr}$-Co-Mo presenta una excelente resistencia a la corrosión. Sin embargo, parece en la actualidad que para dicho tipo de aplicación estructural es la aleación Ti-6Al-4V la que ofrece un mejor potencial debido a sus buenas características mecánicas, su resistencia a corrosión y corrosión-fatiga, su baja densidad, su relativamente bajo módulo elástico y su excelente biocompatibilidad. Puede decirse así mismo que su utilización es general en todos los demás tipos de prótesis articulares. Cabe señalar aquí el posible potencial de la aleación Ti-6A1$7 \mathrm{BNb}$ que se ha citado anteriormente. Esta aleación presenta propiedades y microestructura muy parecidas a la Ti-6A 1-4V y sólo puede considerarse que presenta una biocompatibilidad mejorada, aunque no demostrada, en relación a ésta.

Probablemente el principal problema de las prótesis articulares está en su fijación al hueso vivo. Deben señalarse dos problemas de gran importancia aquí: en primer lugar, la diferencia de módulo elástico entre el metal y el hueso, y en segundo lugar la encapsulación fibrosa que el organismo generará entorno al metal.

Todo ello irá en detrimento de una buena fijación y promoverá la existencia de una intercara viscoelástica y con ello el movimiento relativo entre las superficies ósea y metálica. Todo ello ha llevado a la necesidad de intentar crear una intercara bioactiva en la superficie metálica que desencadene una actividad osteogenética en el hueso y se produzca así un firme anclaje de la prótesis al hueso circundante.

Como se ha mencionado más arriba, la técnica de Charnley utiliza cemento óse a base de polimetilmetacrilato. El cemento crea dos intercaras: metal-cemento y cemento-hueso. La experiencia demuestra que todas las prótesis cementadas acaban aflojándose haciéndose necesario el recambio $(39,40)$. La ventaja del cemento reside en la facilidad de su colocación, la uniforme distribución de cargas de contacto que ejerce, y el menor dolor que sufre el paciente al que se le coloca una prótesis cementada. En pacientes de edad avanzada y de poca actividad se consiguen tiempos de aflojamiento incluso superiores a los quince años. Sin embargo, en pacientes más jóvenes, este tiempo puede llegar a ser incluso inferior a tres años. Es por ello que a pacientes con elevado grado de movilidad se les coloca en general prótesis no cementadas, auque su fijación es por contacto directo metal-hueso y requieren un adecuado mecanizado de la superficie del canal medular femoral. El inconveniente aquí sigue siendo la intercara metal bioinerte-hueso.

Recientemente se han desarrollado nuevos tipos de superficies que permitan inducir una buena actividad 
osteogenética. Entre estas superficies cabe señalar los recubrimientos porosos y los recubrimientos con hidroxiapatito. Los primeros se producen mediante la sinterización de partículas de titanio sobre la superficie que deba estar en contacto con el hueso. En general se buscan tamaños de poro superiores a los $100 \mu \mathrm{m}$, de forma que en dichos poros se produzca crecimiento óseo y se alcance así una buena fijación del implante al hueso (41). Este tipo de superficie porosa es de reciente aplicación tanto en implantes ortopédicos como dentales y no existen todavía estadísticas clínicas que permitan establecer de forma definitiva su mejor comportamiento en relación a las prótesis cementadas y no cementadas. Sin embargo, parecen presentar el problema de una menor vida a fatiga debido al efecto concentrador de tensiones en los poros en contacto con la superficie metálica (42).

Dentro de este mismo contexto cabría presentar las prótesis de rodilla, así como de otras articulaciones. La figura 12 presenta el aspecto de una prótesis de rodilla bicompartimental con recubrimiento poroso de titanio para mejorar su fijación. Los criterios de selección del material y de su estado superficial son los mismos que para las prótesis de cadera, cambiando tan solo los criterios biomecánicos para su diseño.

Por su parte y muy recientemente se han comenzado a comercializar prótesis ortopédicas y dentales de Ti y de Ti-6A1-4V recubiertas de hidroxiapatito. El hidroxiapatito es una de las estructuras que presentan los fosfatos de calcio en el hueso. De hecho y de forma simple se puede decir que el hidroxiapatito es una cerámica constituyente mineral del hueso. Por consiguiente, el organismo reconoce el hidroxiapatito sintético como propio y el hueso puede crecer el contacto con el mismo evitando la formación de una cápsula fibrosa. Esto significa tener una superficie bioactiva que conduce a una excelente fijación prótesis-hueso (43). Este sistema está alcanzando gran aceptación aunque tampoco aquí existen hasta la fecha resultados clínicos estadísticos que permitan valorar adecuadamente sus ventajas. Una de las técnicas de recubrimiento más ampliamente utilizadas es la deposición por plasma del hidroxiapatito sobre las superficies de titanio.

\subsection{IMPLANTES DENTALES.}

Los implantes dentales se utilizan cada vez más ampliamente y principalmente se consideran dos tipos

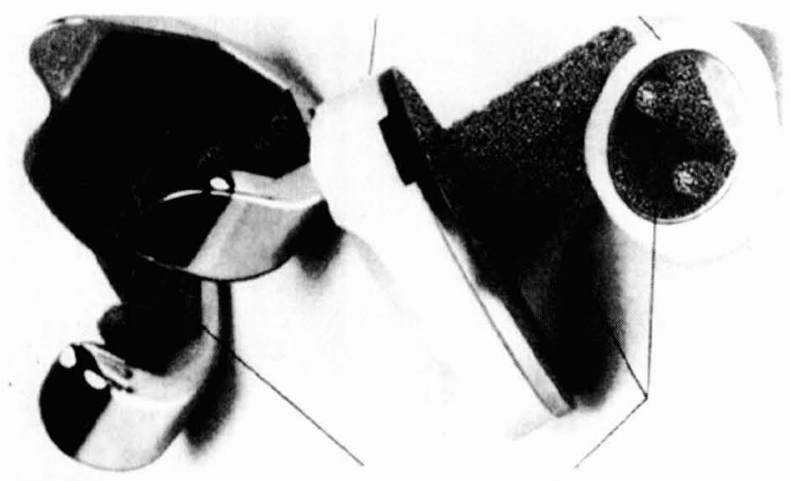

RPCURRTMIENTO POROSO de ellos: los que dan soporte a la dentadura (implantes subeperiostales) y los que substituyen al diente (implantes endóseos). Estos implantes deben tener excelente biocompatibilidad, resistencia a la corrosión, alta resistencia compresiva y tenacidad y la fijación viable entre el hueso alveolar y el tejido mucoso.

En el implante endóseo consiste en alcanzar la fijación del hueso a lo largo de un poste metálico, este se cubrirá con la corona después de que el implante se haya fijado (31). Estos postes pueden ser de acero inoxidable, de Ti-6Al-4V, de titanio o de CoCr. Los que en la actualidad ofrecen mayor potencial son los de Ti y los de Ti-6A1-4V, que a su vez pueden ir recubiertos o no de un recubrimiento poroso o de un recubrimiento de hidroxiapatito.

Otro tipo de implantes dentales son los alambres de ortodoncia, que se utilizan de acero inoxidable AISI 316L y Co-Cr. Recientemente se han empezado a utilizar las aleaciones $\mathrm{Ni}$-Ti aprovechando su superelasticidad. Estas aleaciones pueden alcanzar deformaciones del $10 \%$ a cargas de $600 \mathrm{MPa}$, mientras que las aleaciones convencionales no superan deformaciones elásticas del $2 \%$. Este hecho demuestra que una vez deformado el alambre y colocado en la boca, a una temperatura de $37{ }^{\circ} \mathrm{C}$, la recuperación elástica produce una tensión tendente a corregir la posición de los dientes sobre los que actúa el alambre de ortodoncia $(44,45)$.

\section{AGRADECIMIENTOS}

Los autores del presente trabajo desean agradecer a Industrias Quirúrgicas de Levante S.A. la donación de material para la realización del presente trabajo.

\section{REFERENCIAS}

1. Bothe, R.T., Beaton, K.E. y Davenport, H.A. Surg Gynecol Obstet 1940; 71: 598.

2. Polmear, I.J. «Light alloys: Metallurgy of the light metals». Edward Arnold. $2^{a}$ edición. 1989

3. García Poggio, J.A., Tarín, P. y Suárez, E. «Estudio de las transformaciones estructurales de diversas aleaciones de titanio». VI Asamblea General del CENIM. Madrid 1985, 10.

4. ASTM Bookk of Standards. vol 13.01. Norma F136. 1986; 28-30.

5. Beder, O.E. y Eade, G. Surgery, 1956; 39: 473.

6. Lynch, C.T. «Handbook of Materials Science» vol. 1 (CRC Press, Boca Raton, Florida), 1974; 342-348.

7. Griffin, C.D., Buchanan, R.A. y Lemons, J.E. J Biomed Mat Res, 1983; 18: 489.

8. Collings, E.W. «The physical metallurgy of Titanium Alloys». American Society for Metals. 1984

9. Lewis, R.E., Bjeletich, J.G., Morton, T.M. y Crossley, F.A. Cracks and Fracture, ASTM STP 601, 1976; 371-390.

10. Chesnutt, J.C., Rhodes, C.G. y Williams, J.C. Fractography Microscopic Cracking Processes. ASTM.STP. 600,$1976 ; 99-138$.

11. Tarín P. «El Titanio y sus aleaciones». Curso de Alta Innovación. CEAM, 1990; 18.

Fig. 12. Prótesis de rodilla. 
12. Gil, F.J. y Planell, J.A. Actas de la XXIII Reunión de la Real Sociedad Española de Química. Salamana 1990; 234.

13. Planell, J.A., Catalán, J.J., Vázquez, J.J. y Anglada, M. Tratermat 88, CENIM, Madrid, 1988; 57-76.

14. Eylon, D. y Pierce, C.M. Metall Trans 1976; 7A: $111-121$.

15. Steele, R.K. y McEvily, J. Eng Fract Mec 1976; 8: $31-37$.

16. Yoder, G.R., Cooley, L.A. y Crooker, T.M. Metall Trans, 1977; 8A: 1737-1743.

17. Gil, F.J., Marsal, M., Anglada, M., Vázquez, J.J. y Planell, J.A. $7^{\circ}$ Congreso Nacional de Ciencia y Tecnologías Metalúrgicas. CENIM. Vol II. 1990; 27-35.

18. Gil, F.J., Marsal, M., Anglada, M. y Planell, J.A. Tratermat 90. Zaragoza 1990. Aceptado. Sesión técnica B.

19. Mears, D.C. «Titaniun. Its occurrence Chemistry and Technology». (Ronald Press Co. New York, 1975), 133.

20. Crooker, T.W. y Large, E.A. Amer Soc Test Mater Tech Publ, 1968; 432: 133.

21. Cornet, A., Muster, D. y Jaeger, J.H. Biomat Med Devices Artif Organs, 1979; 7: 155-167.

22. Grover, H.J. J Mater., 1966; 1: 413-424.

23. Williams, D.F. y Roat, R. "Implants in surgery». Saunders Ed. Philadelphia (1973).

24. Gil, F.J. Tesis Doctoral. Universidad de Barcelona (1988).

25. Funkaubo, H. «Shape memory alloys». Gordon and Breach Science Publishers. (1989).

26. Suzuki, Y. Bulletin of Japan Institute of Metals., 1983; 22-8: 730

27. Delaey, L., Derruyterre, J., Aernoudt, E. y Ross, J. INCRA Research Report, 1978; 238: 55-65.
28. Wayman, C.M. Journal of Metals., 1978; 32-6: 121-132.

29. Andreasen, G.F. y Morrow, R.E. American Journal Orthodontics, 1978: 73-2: 142-151.

30. Williams, D.F. J Mater Sci, 1987; 22: 3421-3445.

31. Park, J.B. «Biomaterials Science and Engineering». Plenum Press Ed, 1984; 171-192.

32. Williams, D.F. «Biocompatibility of implant materials». Sector Publishing Limited. London. (1976).

33. Albrektsson, T. Rev Biocompat, 1985; 1: 53-84.

34. Healy, K.E. y Ducheyne, P. 3rd. International Confernce Biointeractions 90. Oxford, 1990; 25.

35. Rieu, J., Pichat, A., Rabbe, L.M., Rambert, A., Chabrol, C. y Robelet, M. 3rd. International Conferenc Biointeractinos 90. Oxford, 1990; 19.

36. Blumm, G.W., Wait, M.E., Walker, P.S. 3rd International Conference Biointeractions 90. Oxford, 1990; 13.

37. Swanson, S.A.V. y Freeman, M.A.R. «The scientific basis of joint replacement». Pitman Medical. (1977).

38. Charnley, J. J Bone It Surg., 1960; 42B: 28.

39. Wilson, J.N. y Scales, J.T. Clin Orthop Rel Res, 1970; 72: 145 .

40. Eftekhar, N.S. Clin Orthop Rel Res., 1987; 225: 207.

41. Boboy, J.D., Pilliar, R.M., Cameron, H.V. y Weatherly. G.C. Clin Orthop Rel Res, 1980; 150: 263-270.

42. Cook, S.D., Thongpreda, N., Anderson, R.C., Hadad. R.J. J of Biomed Mat Res, 1988; 22: 287-302.

43. Klein, C.P.A.T., de Blieck-Hogervost, J.M.A., Wolke, J.G.C. y de Groot, K. «Clinical Implant Materials». Elsevier. Oxford, 1990; 271-276.

44. Takahashi, D.E. J of Dental Eng, 1983; 66: 28-32.

45. Bustone, C.J. Am J of Orthodontics, 1981; 80-1. 